Original Research Paper

\title{
On Fixed Point Theorem of $C$ Class Functions - $B$ Weak Cyclic Mappings
}

\author{
${ }^{1}$ Sahar Mohamed Ali Abou Bakr and ${ }^{2}$ Arslan Hojat Ansari \\ ${ }^{I}$ Department of Mathematics, Faculty of Science, Ain Shams University, Cairo, Egypt \\ ${ }^{2}$ Department of Mathematics, Karaj Branch, Islamic Azad University, Karaj, Iran
}

\author{
Article history \\ Received: 16-04-2017 \\ Revised: 03-07-2017 \\ Accepted: 11-07-2017

\section{Corresponding Author:} \\ Sahar Mohamed Ali Abou Bakr \\ Department of Mathematics, \\ Faculty of Science, Ain Shams \\ University, Cairo, Egypt \\ Email: saharmali@yahoo.com \\ saharmali@sci.asu.edu.eg
}

\section{Introduction}

First; the class $\Phi$ is all non-decreasing mappings $\phi$ : $[0, \infty) \rightarrow[0, \infty)$ characterized by $\phi(t)=0$ if and only if $t=0$.

Bilgili et al. (2014; Karapinar and Sadarangani, 2012; $\mathrm{Du}$ and Karapinar, 2013) discussed the concept of $\Phi$ weakly cyclic contraction mappings and proved fixed point theorems for mappings on Banach spaces. While Harjani et al. (2013) considered cyclic weak $\Phi$ contraction on compact metric spaces, the considered mapping $\phi$ need not be continuous, another fixed point treating the concept is given in (Karapinar and Sadarangani, 2012). The fixed point theorem given in (Karapinar and Sadarangani, 2012) focused on a wider class of metric spaces.

Jleli et al. (2014; Karapinar et al., 2012b) generalized the results to cyclic $(\varphi, \phi)$-weak contractions in some other metric spaces.

Many results have been proved in different situations and settings for the purpose of generalization of the Banach contraction principle for contraction mappings and for non-expansive mappings, (Hardy and Rogers, 1973; Gregus, 1980; Kaewcharoen and Kirk, 2006; Kannan, 1971; Kirk, 1965; Park, 1980; Rhoades, 1977; 2001; Sahar Mohamed Ali Abou Bakr, 2013; Wong, 1975; Rhoades, 2009; Ćirić, 2006).

Recent results related to cyclic weak $(\varphi-\phi)$ contraction mappings appeared in (Sahar Mohamed Ali Abou Bakr, 2017) for mappings with weak cyclic representation in complete metric spaces and weakly complete normed spaces, the considered mapping $\varphi$ need not be additive, the author gave some examples.
On the other side, Morales and Rojas (2009) defined $B Z$ type mappings or the $B$-Zamfirescu mappings, the mappings of any of the following types:

- $B B$-type or $B$-Banach contraction

- $B K$ type or $B$-Kannan contraction

- $B C$ type or $B$-Chatterjea contraction

It is proved the existence of only one fixed point for such types of mapping for the continuous, one to one and sub-sequentially convergent mapping $B$.

For generalized cyclic weak $\varphi-\phi$ contraction types mappings, (Bilgili and Karapinar, 2013; Hussain et al., 2014; Karapinar and Rakocevic, 2013; Karapinar et al., 2012a; 2013).

\section{Mathematical Preliminaries}

First, in the sequel, $(X, d)$ is the space $X$ with a metric $d$ and the class $\mathfrak{U}$ is the class of all finite collections of nonempty closed subsets of $X, \mathfrak{A}=\left\{A_{i}\right\}_{i=1}^{j}$ such that $X=\bigcup_{i=1}^{j} A_{i}:$

$$
\mathfrak{U}:=\left\{\mathfrak{A}=\left\{A_{i}\right\}_{i=1}^{j}: X=\bigcup_{i=1}^{j} A_{i}\right\}
$$

The mappings $B$ and $S$ are self mappings on $X$. We have the following definitions.

\section{Definition 1 (Bilgili and Karapinar, 2013)}

$B$ is known as weak $\Phi$-contraction if and only if there is a continuous function $\phi \in \Phi$ such that: 


$$
d(B(x), B(y)) \leq d(x, y)-\phi(d(x, y)) \text { for every } x, y \in X .
$$

\section{Definition 2 (Bilgili et al., 2014)}

The element $\mathfrak{A}=\left\{A_{i}\right\}_{i=1}^{j} \in \mathfrak{U}$ is $B$ cyclic representation of $X$ if and only if:

$$
B\left(A_{1}\right) \subset A_{2}, B\left(A_{2}\right) \subset A_{3}, \ldots, B\left(A_{j-1}\right) \subset A_{j} \text { and } B\left(A_{j}\right) \subset A_{1}
$$

\section{Definition 3 (Jleli et al., 2014)}

$B$ is $\mathfrak{U}$-cyclic $\Phi$-weak contraction on $X$ if and only if there are $\mathfrak{A} \in \mathfrak{U}$ and a continuous function $\phi \in \Phi$ satisfying the two conditions:

- $\quad A$ is a $B$ cyclic representation of $X$

- $\quad d(B(x), B(y)) \leq d(x, y)-\phi(d(x, y))$

for every $x \in A_{k}, y \in A_{k+1}, k=1,2, \ldots, j$ and $A_{j+1}=A_{1}$.

\section{Definition 4}

$B$ is U-cyclic $\varphi$ - $\phi$-weak contraction on $X$ if and only if there are $\mathfrak{A} \in \mathfrak{U}$ and $\varphi, \phi \in \Phi$, with $\varphi$ continuous such that the following are true:

- $\quad \mathfrak{A}$ is $B$ cyclic representation of $X$

- $\varphi(d(B(x), B(y))) \leq \varphi(d(x, y))-\phi(d(x, y))$

$\forall x \in A_{k} ; y \in A_{k+1}, k=1,2, \ldots, j$ and $A_{j+1}=A_{1}$

This paper generalizes the $\mathfrak{U}$-cyclic $\varphi$ - $\phi$-weak contraction types to new $B$-U-d-cyclic weak $F-\varphi-\phi$ contraction type, this new $C$-class of weak contraction mapping is defined step by step next.

\section{Definition 5}

Let $\mathfrak{A}=\left\{A_{i}\right\}_{i=1}^{j}$ be an element in $\mathfrak{U}$. Then $\mathfrak{A}$ is $B S$ cyclic representation of $X$ if and only if:

$$
\begin{aligned}
& B\left(S\left(A_{1}\right)\right) \subset A_{2}, B\left(S\left(\left(A_{2}\right)\right)\right) \subset A_{3}, \ldots, \\
& B\left(S\left(A_{j-1}\right)\right) \subset A_{j} \text { and } B\left(S\left(\left(A_{j}\right)\right)\right) \subset A_{1}
\end{aligned}
$$

\section{Definition 6}

Let $\mathfrak{A}=\left\{A_{i}\right\}_{i=1}^{j} \in \mathfrak{U}$. Then the self mapping $S$ on $X$ is $B$-cyclic weak $\varphi-\phi$ contraction mapping on $X$ if and only if there are $\varphi, \phi \in \Phi$ with $\varphi$ continuous such that the following are true:

- $\quad \mathfrak{A}$ is a $T S$-cyclic representation of $X$
- $\quad \varphi(d(B(S(x)), B(S(y)))) \leq \varphi(d(B(x), B(y)))-\phi(d(B(x), B(y)))$ $\forall x \in A i, y \in A_{i+1}, i=1,2, \ldots, j$ and $A_{j+1}=A_{1}$

\section{Remark}

The weak type contraction mapping defined in (6) generalizes the definition of cyclic weak $\phi$-contraction of Erdal Karapinar, Kishin Sadarangani, cyclic weak $(\varphi-\phi)$ contraction of Sahar Mohamed Ali Abou Bakr and $T B$ contraction mappings of Jose R. Morales, Edixon Rojas (Karapinar and Sadarangani, 2012; Sahar Mohamed Ali Abou Bakr, 2017; Morales and Rojas, 2009) respectively, because these are a particular cases corresponding to taking $B$ and $\varphi$ identities.

\section{Definition 7}

$B$ is sequentially convergent if it satisfies the condition: If $\left\{B\left(x_{n}\right)\right\}_{n=1}^{\infty}$ is convergent, then $\left\{x_{n}\right\}_{n=1}^{\infty}$ is convergent.

Finally, we have the following:

Definition 8 (Ansari, 2014; Ansari et al., 2016)

The real valued mapping $F:[0, \infty) \times[0, \infty) \rightarrow R$ is $C$ class if it is continuous and satisfying the axioms:

- $F(u, v) \leq u$ for all $u, v \in[0 ; \infty]$

- If $F(u ; v)=u$, then either $u=0$ or $v=0$

$C$ is the set of $C$-class functions. Mention that some $C$-class function $F$ verifies $F(0,0)=0$.

\section{Examples}

If $h:[0, \infty) \rightarrow[0, \infty)$ is a continuous and $h(v)=0$ if and only if $v=0$, then $F(u, v)=u-h(v)$ is a $C$-class function, in particular:

- $\quad F(u, v)=u-v$

- $F(u, v)=u-\frac{v}{k+v}$

- $F(u, v)=u-\left(\frac{2+v}{1+v}\right) v$

- $F(u, v)=u-\left(\frac{1+u}{2+u}\right)\left(\frac{v}{1+v}\right)$

are $C$-class functions.

If $h:[0, \infty) \rightarrow[0,1]$ is a continuous function, then $F(u, v)=u h(v)$ is a $C$-class function, in particular we have the following:

- $\quad F(u, v)=m u$ for some $m \in[0,1]$

- $F(u, v)=\frac{u}{(1+v)^{r}}$ for some $r \in[0, \infty)$ 
- $\quad F(u, v)=u \log _{v+a} a, a>1$

- $F(u, v)=\frac{u}{(1+u)^{r}}$ for some $r \in[0, \infty)$

In addition to the following:

- $\quad F(u, v)=\log \left(v+a^{u}\right) /(1+v)$, for some $a>1$

- $\quad F(u, v)=\ln \left(1+a^{u}\right) / 2$, for $e>a>1$. Indeed $F(u, v)=u$ implies that $u=0$

- $\quad F(u, v)=(u+l)^{\left(1 /(1+v)^{r}\right)}-l, l>1$, for $r \in[0, \infty)$

- $F(u, v)=\sqrt[n]{\ln \left(1+u^{n}\right)}$

Now; the generalized $C$-class of $T S$ cyclic weak $(\varphi$, $\phi)$-contraction mappings are defined as:

\section{Definition 9}

$S$ is $\mathfrak{U}$ - $B$-cyclic $F-\varphi$ - $\phi$-weak contraction mapping on $X$ if and only if there are $\mathfrak{A}=\left\{A_{i}\right\}_{i=1}^{j} \in \mathfrak{U}, \varphi, \phi \in \Phi$ with $\varphi$ continuous and $F \in C$ satisfying:

- $\quad \mathfrak{A}$ is a $B S$-cyclic representation of $X$

$$
\begin{aligned}
& \varphi(d(B(S(x)), B(S(y)))) \\
& \leq F(\varphi(d(B(x), B(y))), \phi(d(B(x), B(y))))
\end{aligned}
$$

for every $x \in A_{k}, y \in A_{k+1}, k=1,2, \ldots, j$ and $A_{j+1}=A_{1}$

\section{Remark}

The contraction type mapping defined in definition (9) is a generalization of the contraction type defined in definition (6), because it is a particular case when taking $F(u, v)=u-v$.

\section{Main Results}

The results of this work are depending on Propositions (1) and (2) below.

\section{Proposition 1}

Let $S$ be $\mathfrak{U}-B$-cyclic $F-\varphi$ - $\phi$-weak contraction on $X$. Then:

$$
\inf \{d(B(S(x)), B(x)): x \in X\}=0
$$

\section{Proof}

Choose $x_{0} \in X$ and focus on the iterated sequence:

$$
B\left(x_{n+1}\right)=B\left(S\left(x_{n}\right)\right)=B\left(S^{n}\left(x_{0}\right)\right) \text { for } n=0,1,2, \ldots
$$

If there is a natural number $n_{0}$ such that $S\left(x_{n+1}\right)=$ $S\left(x_{n}\right) \forall n \geq n_{0}$, then $S\left(x_{n}\right)$ is fixed of $S$. This insures that such infimum is zero. Suppose that $B\left(S\left(x_{n+1}\right)\right) \neq$ $B\left(S\left(x_{n}\right)\right)$ for all $n=0,1,2, \ldots$ Then, the contraction condition yields:

$$
\begin{aligned}
& \varphi\left(d\left(B\left(x_{n}\right), B\left(x_{n+1}\right)\right)\right)=\varphi\left(d\left(B S\left(x_{n-1}\right), B\left(S x_{n}\right)\right)\right) \\
& \leq F\left(\varphi\left(d\left(B\left(x_{n-1}\right), B\left(x_{n}\right)\right)\right)=\phi\left(d\left(B\left(x_{n-1}\right), B\left(x_{n}\right)\right)\right)\right) \\
& \leq \varphi\left(d\left(B\left(x_{n-1}\right), B\left(x_{n}\right)\right)\right)
\end{aligned}
$$

Since $\varphi$ is non-decreasing function, we see that:

$$
d\left(B\left(x_{n}\right), B\left(x_{n+1}\right)\right) \leq d\left(B\left(x_{n-1}\right), B\left(x_{n}\right)\right) \forall n \in N
$$

This proves that the sequence $\left\{d\left(B\left(x_{n}\right), B\left(x_{n+1}\right)\right)\right\}_{n \in N}$ is a non-decreasing, hence the limit:

$$
\lim _{n \rightarrow \infty} d\left(B\left(x_{n}\right), B\left(x_{n+1}\right)\right)
$$

exists and it is equal to the infimum of the sequence, say $r$ :

$$
\begin{aligned}
& r=\lim _{n \rightarrow \infty} d\left(B\left(x_{n}\right), B\left(x_{n+1}\right)\right)=\inf \left\{d\left(B\left(x_{n}\right), B\left(x_{n+1}\right)\right): n \in N\right\}, \\
& r \leq d\left(B\left(x_{n}\right), B\left(x_{n+1}\right)\right) \forall n \in N
\end{aligned}
$$

On the other side, we have the same conclusions for the two sequences $\left\{\varphi\left(d\left(B\left(x_{n}\right), \quad B\left(x_{n+1}\right)\right)\right)\right\}_{n \in N}$ and $\left\{\phi\left(d\left(B\left(x_{n}\right), B\left(x_{n+1}\right)\right)\right)\right\} n \in N$, consider the two positive real numbers $\phi_{0}$ and $\varphi_{0}$ given as follows:

$$
\begin{aligned}
& \phi(r) \leq \phi_{0}=\lim _{n \rightarrow \infty} \phi\left(d\left(B\left(x_{n}\right), B\left(x_{n+1}\right)\right)\right) \\
& =\inf \left\{\phi\left(d\left(B\left(x_{n}\right), B\left(x_{n+1}\right)\right)\right): n \in N\right\}
\end{aligned}
$$

and:

$$
\begin{aligned}
& \varphi(r) \leq \varphi_{0}=\lim _{n \rightarrow \infty} \varphi\left(d\left(B\left(x_{n}\right), B\left(x_{n+1}\right)\right)\right) \\
& =\inf \left\{\varphi\left(d\left(B\left(x_{n}\right), B\left(x_{n+1}\right)\right)\right): n \in N\right\}
\end{aligned}
$$

The limit of the inequalities (3.1) as $n \rightarrow \infty$ gives:

$$
\varphi_{0} \leq F\left(\varphi_{0}, \lim _{n \rightarrow \infty} \phi\left(d\left(B\left(x_{n-1}\right), B\left(x_{n}\right)\right)\right)\right) \leq \varphi_{0}
$$

and, therefore:

$$
\varphi_{0}=0, \text { or } \lim _{n \rightarrow \infty} \phi\left(d\left(B\left(x_{n-1}\right), B\left(x_{n}\right)\right)\right)
$$


Now; suppose that $r>0$, we have $\phi(r)>0$, thus:

$$
0<\phi(r) \leq \phi\left(d\left(B\left(x_{n-1}\right), B\left(x_{n}\right)\right)\right) \forall n \in N
$$

Letting $n \rightarrow \infty$ in the last inequalities, we get the following contradiction:

$$
0<\phi(r) \leq \lim _{n \rightarrow \infty} \phi\left(d\left(B\left(x_{n-1}\right), B\left(x_{n}\right)\right)\right)=0
$$

that is; the assumption $r>0$ is not true, hence $r=0$, thus:

$$
\begin{aligned}
& \lim _{n \rightarrow \infty} d\left(B\left(x_{n+1}\right), B\left(x_{n}\right)\right) \\
& =\inf \left\{d\left(B\left(x_{n+1}\right), B\left(x_{n}\right)\right): n \in N\right\}=0
\end{aligned}
$$

Hence; $\left\{x_{n}\right\}_{n=1}^{\infty} \subset X$ such that:

$$
\lim _{n \rightarrow \infty} d\left(B\left(S\left(x_{n}\right)\right), B\left(x_{n}\right)\right)=0
$$

This is sufficiently proved that $\inf \{d(B(S(x)), B(x))$ : $x \in X\}=0$.

\section{Proposition 2}

Let $S$ be $\mathfrak{U}$ - $B$-cyclic $F$ - $\varphi$ - $\phi$-weak contraction mapping on $X$. Then the iterated sequence $\left\{B\left(x_{n}\right)=B\left(S^{n}\left(x_{0}\right)\right)\right\}_{n \in N}$ is Cauchy.

\section{Proof}

We determine for a given $\epsilon>0$ a natural number $n_{0} \in N$ satisfying; if $m, n>n_{0}$ with $x_{n} \in A_{i}$ and $x_{m} \in A_{i+1}$ for some $i \in\{1,2, \ldots j\}(n-m \equiv 1(j))$ then $d\left(B\left(x_{n}\right), B\left(x_{m}\right)\right)<\epsilon$ gives a contradiction.

Suppose that there is $\epsilon>0$ satisfying; any $n_{0} \in N$ yields $m, n>n_{0}$ with $B\left(x_{n}\right) \in A_{i}$ and $B\left(x_{m}\right) \in A_{i+1}$ for some $i \in\{1$, $2, \ldots, j\}(n-m \equiv 1(j))$ satisfying:

$$
\epsilon<d\left(B\left(x_{n}\right), B\left(x_{m}\right)\right)
$$

since $\varphi$ and $\phi$ are non-decreasing, we see that:

$$
\begin{aligned}
& 0<\varphi(\epsilon) \leq \varphi\left(d\left(B\left(x_{n}\right), B\left(x_{m}\right)\right)\right) \\
& \text { and } \\
& 0<\phi(\epsilon) \leq \phi\left(d\left(B\left(x_{n}\right), B\left(x_{m}\right)\right)\right)
\end{aligned}
$$

and since $\varphi$ is continuous, we see that:

$$
0<\varphi(\epsilon) \leq \varphi\left(\lim _{n, m \rightarrow \infty}\left(d\left(B\left(x_{n}\right), B\left(x_{m}\right)\right)\right)\right)
$$

using the contractivity condition of $S$ we see that:

$$
\begin{aligned}
& \varphi\left(d\left(B\left(x_{n+1}\right), B\left(x_{m+1}\right)\right)\right) \\
& =\varphi\left(d\left(B\left(S^{n+1}\left(x_{0}\right)\right), B\left(S^{m+1}\left(x_{0}\right)\right)\right)\right) \\
& \leq F\left(\begin{array}{l}
\varphi\left(d\left(B\left(S^{n}\left(x_{0}\right)\right), B\left(S^{m}\left(x_{0}\right)\right)\right)\right) \\
\phi\left(d\left(B\left(S^{n}\left(x_{0}\right)\right), B\left(S^{m}\left(x_{0}\right)\right)\right)\right)
\end{array}\right) \\
& \leq \varphi\left(d\left(B\left(x_{n}\right), B\left(x_{m}\right)\right)\right)
\end{aligned}
$$

Now; using inequalities (3.2), (3.3) and letting $n$, $m \rightarrow \infty$ in (3.4) with $n-m \equiv 1(j)$ proves the following contradiction:

$$
\varphi(\epsilon) \leq F(\varphi(\epsilon), \phi(\epsilon)) \leq \varphi(\epsilon)
$$

This proves that $F(\varphi(\epsilon), \phi(\epsilon))=\varphi(\epsilon)$, hence either $\varphi(\epsilon)$ $=0$ or $\phi(\epsilon)=0$, consequently $\epsilon=0$, therefore this sequence is having Cauchy subsequence, this fact with Proposition (1) completes the proof.

Finally; we have:

\section{Theorem 1}

If $(X, d)$ is complete, $B$ is one to one continuous self sequentially convergent mapping on $X$ and $S$ is $\mathfrak{U}-B$ cyclic $F-\varphi$ - $\phi$-weak contraction mapping on $X$. Then $S$ owns fixed points. Moreover; we have the following:

- If $\left\{A_{i}\right\}_{i=1}^{j}$ is representation of $X$ and $z$ is fixed of $S$, then $B(z) \bigcap_{i=1}^{j} A_{i}$

- Arbitrarily two consecutive sets of $\left\{A_{i}\right\}_{i=1}^{j}$ cannot contain two different fixed points

\section{Proof}

Using Proposition (2), the sequence $\left\{B\left(S^{n}\left(x_{0}\right)\right)\right\}_{n \in N}$ is Cauchy, the completeness of $X$ shows that there is a point $y \in X$ such that:

$$
\lim _{n \rightarrow \infty} B\left(S^{n}\left(x_{0}\right)\right)=y
$$

Such a limit point is lying in the set $\bigcap_{i=1}^{j} A_{i}$, because each $A_{i}, i=1,2, \ldots, j$ contains in finitely many members of the infinite sequence $\left\{B\left(S^{n}\left(x_{0}\right)\right)\right\}_{n \in N}$, hence $y$ is a limit point for $A i$ for each $i=1,2, \ldots, j$, giving that $A i$ is closed for each $i=1,2, \ldots, j$ shows that $y \in A_{i}$ for each $i=1,2, \ldots, j$ (as closed set contains all its limit points), thus:

$$
y \in \bigcap_{i=1}^{j} A_{i}
$$


Since $B$ is sequentially convergent mapping, the sequence $\left\{S^{n}\left(x_{0}\right)\right\}_{n=1}^{\infty}$ has a convergent subsequence $\left\{S^{n_{k}}\left(x_{0}\right)\right\}_{n=1}^{\infty}$, hence there is $z \in X$ such that:

$$
\lim _{k \rightarrow \infty} S^{n_{k}}\left(x_{0}\right)=z
$$

Using the continuity of $B$ gives that:

$$
\lim _{k \rightarrow \infty} B\left(S^{n_{k}}\left(x_{0}\right)\right)=B(z)
$$

Using (3.6) and (3.7) proves that $B(z)=y$. We will see that such a $z$ is fixed of $S$. In fact; there is $i \in\{1$, $2, \ldots, j\}$ such that $z \in A_{i}$, for the number $i+1$ we get $n_{i} \in N$ such that:

$$
\left\{x_{n_{i}}, x_{n_{i}+1}, x_{n_{i}+2}, \ldots\right\} \subset A_{i+1} .
$$

Therefore $z$ and $\left\{x_{n_{i}}, x_{n_{i}+1}, x_{n_{i}+2}, \ldots\right\}$ are lying in two consecutive sets, on the other side we have:

$$
\begin{aligned}
& d(B(S(z)), B(z)) \leq d\left(B(S(z)), B\left(x_{n_{i+1}}\right)\right) \\
& +d\left(B\left(x_{n_{t+1}}\right), B(z)\right) \\
& =d\left(B(S(z)), B\left(S\left(x_{n_{i}}\right)\right)\right)+d\left(B\left(S\left(x_{n_{i}}\right)\right), B(z)\right) .
\end{aligned}
$$

Hence we use the contraction property of $S$ as:

$$
\begin{aligned}
& \varphi\left(d\left(B(S(z)), B\left(S\left(x_{n_{i}}\right)\right)\right)\right. \\
& \leq F\left(\varphi\left(d\left(B(z), B\left(x_{n_{i}}\right)\right)\right), \phi\left(d\left(B(z), B\left(x_{n_{i}}\right)\right)\right)\right) \\
& \leq \varphi\left(d \left(B(z), B\left(x_{n_{i}}\right)\right.\right. \\
& =\varphi\left(d\left(B(z), B\left(S\left(x_{n_{i}}\right)\right)\right)\right)
\end{aligned}
$$

Using the continuity of $\varphi$ and taking the limit of the inequalities (3.9) as $i \rightarrow \infty$ prove the following:

$$
\begin{aligned}
& \lim _{i \rightarrow \infty} \varphi\left(d\left(B(S(z)), B\left(S\left(x_{n_{i}}\right)\right)\right)\right. \\
& \leq \lim _{i \rightarrow \infty} \varphi\left(d\left(B(z), B\left(S\left(x_{n_{i}-1}\right)\right)\right)\right) \\
& =\varphi\left(d\left(B(z), \lim _{i \rightarrow \infty} B\left(S\left(x_{n_{i}-1}\right)\right)\right)\right) \\
& =\varphi(d(B(z), y)) \\
& =\varphi(d(B(z), B(z)))=\varphi(0)=0
\end{aligned}
$$

Using the continuity of $\varphi$ once more with the inequalities (3.10) shows that:

$$
\begin{aligned}
& 0=\lim _{i=\infty} \varphi\left(d\left(B(S(z)), B\left(S\left(x_{n_{i}}\right)\right)\right)\right. \\
& =\varphi\left(\lim _{i=\infty} d\left(B(S(z)), B\left(S\left(x_{n_{i}}\right)\right)\right)\right.
\end{aligned}
$$

hence:

$$
\lim _{i=\infty} d\left(B(S(z)), B\left(S\left(x_{n_{i}}\right)\right)\right)=0
$$

Using (3.11) in (3.8) after taking the limit as $i \rightarrow \infty$ gives:

$$
\begin{aligned}
& d(B(S(z)), B(z)) \leq \lim _{i \rightarrow \infty} d\left(B(S(z)), B\left(S\left(x_{n_{i}}\right)\right)\right) \\
& +\lim _{i \rightarrow \infty} d\left(B\left(S\left(x_{n_{i}}\right)\right), B(z)\right) \\
& =0+d(y, B(z))=d(B(z), B(z))=0
\end{aligned}
$$

Hence $d(B(S(z)), B(z))=0$, therefore, $B(S(z))=$ $B(z)$, since $B$ is one to one, we get $S(z)=z$ and hence $z$ is fixed of $S$.

To show that two consecutive sets of $\left\{A_{i}\right\}_{i=1}^{j}$ cannot contain two different fixed points, by contrary assume that $w$ and $z$ are two different fixed of $S, S(w)=w$ and $S(z)=z$ those are lying in two consecutive sets, we have the following:

$$
\begin{aligned}
& \varphi(d(B(w), B(z)))=\varphi(d(B(S(w)), B(S(z)))) \\
& \leq F(\varphi(d(B(w), B(z))), \phi(d(B(w), B(z))))
\end{aligned}
$$

hence $\varphi(d(B(w), B(z)))=0$, or $\phi(d(B(w), B(z)))=0$, that is; $d(B(w), B(z))=0$, consequently $B(w)=B(z)$, since $B$ is one to one, $w=z$. This completes the proof.

We also have:

\section{Theorem 2}

Let $(X,\|\|$.$) be weakly complete normed space, C$ be a closed convex subset of $X,\left\{C_{i}\right\}_{i=1}^{j}$ be a nonempty closed subsets of $C, C=\bigcup_{i=1}^{j} C_{i}$. If $B$ and $S$ are self mappings on $C,\left\{C_{i}\right\}_{i=1}^{j}$ is $B S$ representation of $C$ and $S$ is weak $F-\varphi-\phi$ contraction on $C$, then $S$ has fixed points.

\section{Proof}

Using Proposition (2) the sequence of iterates $\left\{B\left(S^{n}\left(x_{0}\right)\right)\right\}_{n \in N}$ is Cauchy, using the weak completeness assumption of $X$ there exists $x \in X$ such that: 


$$
w-\lim _{n \rightarrow \infty} B\left(S^{n}\left(x_{0}\right)\right)=x
$$

Since $C$ is closed convex subset of $X$, the sequence $\left\{B\left(S^{n}\left(x_{0}\right)\right)\right\}_{n \in N}$ converges strongly to $x$ and $x \in C$. For the other parts of the proof use Theorem (1).

\section{Conclusion}

This paper suggests new $C$-class of $T S$ cyclic weak $(\varphi, \phi)$-contraction mappings and proved the existence of unique fixed point for such types of mappings.

\section{Competing Interest}

The authors have no competing interest.

\section{Author's Contributions}

The first author contributed in the ration of two-third in the writing of this paper.

\section{References}

Ansari, A.H., 2014. Note on $(\varphi-\psi)$-contractive type mappings and related fixed point. Proceedings of the 2nd Regional Conference on Mathematics and Applications, (CMA' 14), Payame Noor University, pp: 377-380.

Ansari, A.H., P. Kumam and B. Samet, 2016. A fixed point problem with constraint inequalities via an implicit contraction. J. Fixed Point Theory Applic., 19: $1145-1163$.

DOI: $10.1007 / \mathrm{s} 11784-016-0320-1$

Bilgili, N. and E. Karapinar, 2013. Cyclic contractions via auxiliary functions on G-metric spaces. Fixed Point Theory Applic., 2013: 49-49. DOI: 10.1186/1687-1812-2013-49

Bilgili, N., I.M. Erhan, E. Karapinar and D. Turkoglu, 2014. Cyclic contractions and related fixed point theorems on g-metric spaces. Applied Math. Inform. Sci., 8: 1541-1551. DOI: 10.12785/amis/080407

Ćirić, L.B., 2006. Contractive type non-self mappings on metric spaces of hyperbolic type. J. Math. Anal. Applic., 317: 28-42.

DOI: $10.1016 /$ j.jmaa.2005.11.025

Du, W.S. and E. Karapinar, 2013. A note on caristi-type cyclic maps: Related results and applications. Fixed Point Theory Applic., 2013: 344-344. DOI: $10.1186 / 1687-1812-2013-344$

Gregus, M., 1980. A fixed point theorem in bannach spaces. Boll. Unione Math. Italiana, 17: 193-198.

Hardy, G.E. and T.D. Rogers, 1973. A generalization of a fixed point theorem of Reich. Canad. Math. Bul., 16: 201-206. DOI: 10.4153/CMB-1973-036-0
Harjani, J., B. Lopez and K. Sadarangani, 2013. Fixed point theorems for cyclic weak contractions in compact metric spaces. J. Nonlinear Sci. Applic., 6: 279-284.

Hussain, N., E. Karapinar, S. Sedghi, N. Shobe and S. Firouzian, 2014. Cyclic $\phi$-contractions in uniform spaces and related fixed point results. Abs. Applied Anal., 2014: 976859-976859.

DOI: $10.1155 / 2014 / 976859$

Jleli, M., E. Karapinar and B. Samet, 2014. On cyclic ( $\psi$, $\phi)$-contractions in Kaleva-Seikkala's type fuzzy metric spaces. J. Intell. Fuzzy Syst., 27: 2045-2053. DOI: $10.3233 /$ IFS-141170

Kaewcharoen, A. and W.A. Kirk, 2006. Nonexpansive mappings defined on unbounded domains. Fixed Point Theory Applic., 2006: 82080-82080. DOI: $10.1155 /$ FPTA/2006/82080

Kannan, R., 1971. Some results on fixed points III. Fundamenta Math., 70: 169-177.

Karapinar, E. and K. Sadarangani, 2012. Corrigendum to "Fixed point theory for cyclic weak $\phi$-contraction". Applied Math. Lett. 25: 1582-1584. DOI: $10.1016 /$ j.aml.2011.11.001

Karapinar, E. and V. Rakocevic, 2013. On cyclic generalized weakly C-contractions on partial metric spaces. Abs. Applied Anal., 2013: 831491-831491. DOI: $10.1155 / 2013 / 831491$

Karapinar, E., G. Petruel and K. Ta, 2012a. Best proximity point theorems for KT-types cyclic orbital contraction mappings. Fixed Point Theory, 13: 537-546.

Karapinar, E., M. Jleli and B. Samet, 2012b. Fixed point results for almost generalized cyclic $(\psi, \phi)$-weak contractive type mappings with applications. Abs. Applied Anal., 2012: 917831-917831. DOI: $10.1155 / 2012 / 917831$

Karapinar, E., S. Romaguera and K. Tas, 2013. Fixed points for cyclic orbital generalized contractions on complete metric spaces. Cent. Eur. J. Math., 11: 552-560. DOI: $10.2478 / \mathrm{s} 11533-012-0145-0$

Kirk, W.A., 1965. A fixed point theorem for mappings which do not increase distances. Am. Math. Monthly, 72: 1004-1006. DOI: 10.2307/2313345

Morales, J.R. and E. Rojas, 2009. Some results on Tzamfirescu operators. Revista Notas de Matematica, 5: 64-71.

Park, S., 1980. On general contraction-type conditions. J. Korean Math. Soc., 17: 131-140.

Rhoades, B.E., 1977. A comparison of various definitions of contractive mappings. Trans. Am. Math. Soc., 226: 257-290. DOI: 10.1090/S0002-9947-1977-0433430-4

Rhoades, B.E., 2001. Some theorems on weakly contractive maps. Nonlinear Anal., 47: 2683-2693. DOI: $10.1016 / \mathrm{S} 0362-546 \mathrm{X}(01) 00388-1$ 
Rhoades, B.E., 2009. Necessary and sufficient condition for common fixed point theorems. J. Adv. Math. Stud.

Sahar Mohamed Ali Abou Bakr., 2017. Fixed point theorem of weak cyclic contraction types of operators. Int. J. Math. Stat.
Sahar Mohamed Ali Abou Bakr., 2013. Fixed point theorems of some type contraction mappings. Int. J. Nonlinear Anal. Convex Anal., 14: 331-342.

Wong, C.S., 1975. On Kannan maps. Proc. Am. Math. Society, 47: 105-116.. 